\title{
Significant Physiological Features to Identify High Performance States
}

\author{
Mariola Peláez ${ }^{1,2}, \mathrm{M}^{\mathrm{a}}$ Teresa Lozano ${ }^{1,3}$, Montserrat Aiger ${ }^{1}$, Alberto Hernando ${ }^{1,2}$, Eduardo Gil $^{2}$ \\ ${ }^{1}$ Centro Universitario de la Defensa (CUD), Zaragoza, Spain \\ ${ }^{2}$ BSICoS Group, I3A, IIS Aragón, CIBER, University of Zaragoza, Spain \\ ${ }^{3}$ RoPeRT Group, Aragón Institute of Engineering Research (I3A), Zaragoza, Spain
}

\begin{abstract}
Our long-term goal is the development of an automatic identifier of attentional states. In order to accomplish it, we should firstly be able to identify different states. So, the first aim of this work is to identify the most appropriate features, to detect a subject high performance state. For that, a database of electrocardiographic (ECG) signal of two unequivocally defined states (rest and attention task) is needed. To achieve this goal, ECG signal is recorded, in those cognitive states from up to 54 subjects as a sample of the population.

Temporal and frequency parameters of heart rate variability have been computed from ECG signal. Additionally, the respiratory rate has been estimated from the same signal. In total, ten features are obtained for each subject. They provide information about the physiological response of the subject and about his autonomic nervous system. Results show that eight from these features present significant differences between subject's baseline and subject's attentional state; and selecting only four of them, state classification accuracy reaches a mean of $75.91 \%$.
\end{abstract}

\section{Introduction}

Attention of security and defense personnel may be affected, among others, by psychological stress and by states of sleep deprivation $[1,2]$. It is also essential for such personnel, as well as for pilots in general, to maintain an adequate divided attention. It is the ability to respond to at least two tasks at the same time [3]. Divided attention is mainly addressed to the study of the deficit as a consequence of the simultaneous presentation of information or the concurrent realization of several activities. Brief Test of Attention (BTA) is standardized for the measure of divided attention [4].

The technological challenge we face is based on the fundamental hypothesis that during the execution of an activity that requires the subject sustained attention, there are alterations in the Autonomic Nervous System (ANS), which can be noninvasively quantified by the recording of physiological signals. These alterations in the ANS can be studied by analyzing the Heart Rate Variability (HRV) from the ECG signal. HRV (and the balance between its two branches: sympathetic and parasympathetic) is one of the most widely used noninvasive measures to evaluate ANS activity [5].

One essential point before the execution of tasks where safety is involved, is to know the person capability to maintain an adequate level of attention at this precise moment. In order to be able to distinguish it, firstly we try to identify the most appropriate physiological variables to detect a high performance subject state. To achieve this goal, an ECG signal database is recorded from up to 54 subjects as a sample of the population. The ECG signal is recorded in two unequivocally defined states: rest and high level of attention. The HRV is estimated from the ECG signal, and classical temporal and frequency parameters are extracted. Also the respiratory rate is estimated from the ECG signal. For each subject, 10 features are obtained, which will provide information about the subject's physiological response and ANS.

\section{Materials and methodology}

\subsection{Data base}

The generated database includes recordings of 54 subjects (50 males and 4 females). Their age is $30.94 \pm 6.19$ years and they are meanly military personnel ( 49 out of 54 ; $91 \%$ of total population).

Subjects' ECG signal was recorded, in order to study differences in biological signals related to two stages:

- Baseline $(B L)$. Subjects remain 5 minutes seated, without performing any task. First 30 seconds from the baseline are discarded. Next 2 minutes are processed.

- Attention test (BTA). During the attention state, subjects perform the Brief Test of Attention (BTA). The BTA test consists of two parts. In the first one, subjects listen to 10 lists of letters and numbers with a variable length between 4 and 18 elements, and write how many numbers each list contains, ignoring the letters. In the second part the 10 lists 
are read again, but this time subjects must count letters, ignoring numbers. Two minutes of the BTA test segment while subjects count numbers are processed.

Recordings of ECG were obtained thanks to Nautilus, a device developed by the University of Kaunas, Lithuania [6]. It allows us to record the ECG signal with three nonorthogonal leads at a sampling frequency of $2000 \mathrm{~Hz}$.

\subsection{Heart rate variability signal}

The Time Variant Integral Pulse Frequency Modulation (TVIPFM) model is used to represent the control of the heart rate (HR) by the ANS [7].

First, heart beats are detected from the recorded ECG signal using an algorithm based on wavelets [8]. Ectopic beats, missed beats and false detections are identified [9]. Then, an instantaneous heart rate signal $d_{\mathrm{HR}}(n)$, sampled at $4 \mathrm{~Hz}$, is obtained from the beat occurrence time series.

Then, a time-varying mean heart rate, $d_{\mathrm{HRM}}(n)$, is obtained by low-pass filtering $d_{\mathrm{HR}}(n)$, with a cut off frequency of $0.03 \mathrm{~Hz}$ :

$$
d_{\mathrm{HR}}(n)=\frac{1+\mathfrak{M}(n)}{T(n)} \quad d_{\mathrm{HRM}}(n)=\frac{1}{T(n)}
$$

where $\mathfrak{M}(n)$ represents the modulating signal which carries the information from ANS and $T(n)$ is the mean heart rate, which is considered to be slow-time-variant by this model. HRV and modulating signals are estimated according to:

$$
d_{\mathrm{HRV}}(n)=d_{\mathrm{HR}}(n)-d_{\mathrm{HRM}}(n) \quad \mathfrak{M}(n)=\frac{d_{\mathrm{HRV}}(n)}{d_{\mathrm{HRM}}(n)}
$$

\subsection{Time-frequency analysis of $\mathrm{HRV}$}

From the beat occurrence time series detected, five temporal parameters were computed as the mean of the two minutes selected for each stage (baseline and attention):

- HRM: mean heart rate,

- SDNN: standard deviation of all normal-to-normal (NN) intervals,

- SDSD: standard deviation of differences between adjacent NN intervals,

- RMSSD: square root of the mean of the squares of the successive differences between adjacent NN,

- pNN50: number of pairs of successive NN that differ by more than $50 \mathrm{~ms}$, divided by the total number of $\mathrm{NN}$.

Time-frequency analysis is applied to $\mathfrak{M}(n)$ to characterize the rapid response of ANS to BTA test. The smoothed pseudo Wigner-Ville distribution (SPWVD) is used because it provides better resolution than nonparametric linear methods, independent control of time and frequency filtering, and power estimates with lower variance than parametric methods when rapid changes occur. SPWVD of $\mathfrak{M}(n)$ is computed according to (8), where $n, m$ are time and frequency indexes respectively.

The analytic signal $a_{\mathfrak{M}}(n)$ is defined as $a_{\mathfrak{M}}(n)=$ $\mathfrak{M}(n)+j \cdot \hat{\mathfrak{M}}(n)$, where $\hat{\mathfrak{M}}(n)$ represents the Hilbert transform of $\mathfrak{M}(n)$. The terms $g(n)$ and $h(l)$ are time and frequency smoothing windows, chosen to be Hamming windows whose lengths are $2 \cdot N+1=203$ and $2 \cdot L+1=1025$ samples, respectively [10].

In relation to frequency, four parameters are calculated based on SPWVD as the mean of the two minutes selected for each stage (baseline and attention):

- $P_{\mathrm{LF}}$ : power in the LF band $(0.04-0.15 \mathrm{~Hz})$,

- $P_{\mathrm{HF}}$ : power in the HF band $(0.15-0.4 \mathrm{~Hz})$,

- $P_{\mathrm{LF}_{\mathrm{n}}}$ : power in LF band normalized respect to powers in LF and HF bands: $P_{\mathrm{LF}_{\mathrm{n}}}=\operatorname{mean}\left(P_{\mathrm{LF}}(n)\right) /\left(\operatorname{mean}\left(P_{\mathrm{LF}}(n)\right)+\right.$ $\left.\operatorname{mean}\left(P_{\mathrm{HF}}\right)(n)\right)$,

- $R_{\mathrm{LF} / \mathrm{HF}}$ : ratio between $\mathrm{LF}$ and $\mathrm{HF}$ power: $R_{\mathrm{LF} / \mathrm{HF}}=$ $\operatorname{mean}\left(P_{\mathrm{LF}}(n)\right) / \operatorname{mean}\left(P_{\mathrm{HF}}(n)\right)$.

\subsection{Respiratory information}

Respiratory information can be extracted from ECG using an ensemble of signals $(\geq 1)$ obtained from it. A method for estimating respiratory rate from electrocardiogram signals is presented in [11]. It is based on QRS slopes and R-wave angle [12], which reflect respiration-induced beat morphology variations [13]. The following series are studied as ECG Derived Respiration (EDR) signals: slope between the peak of $\mathrm{Q}$ and $\mathrm{R}$ waves, slope between the peak of $\mathrm{R}$ and $\mathrm{S}$ waves, and the R-wave angle. The method assigns to each beat occurrence the value of its associate QRS slope and R-wave angle. These signals are unevenly sampled, so a resampling at $4 \mathrm{~Hz}$ to standardize them is applied in addition to a mad-based-outlier rejection. Finally a band-pass filter is applied $(0.075-1 \mathrm{~Hz})$.

In this study three leads are registered and three EDR signals are generated for each lead, so nine final signals conform the ensemble to extract respiratory information to the ECG. An algorithm based on [11] is applied over the nine EDR signals $(j=1 \ldots 9)$. For each signal, a power spectrum density $S_{\mathrm{j}, \mathrm{k}}(f)$ is estimated every 5 seconds from the $k^{t h} 40$ seconds length running window by the Welch periodogram, using sub-windows of 12 seconds and $50 \%$ of overlapping. For each $S_{\mathrm{j}, \mathrm{k}}(f)$, the location of the largest peak $f_{\mathrm{p}}(j, k)$ is detected.

Subsequently, a measure of peakness $P_{\mathrm{j}, \mathrm{k}}$ is obtained from $S_{\mathrm{j}, \mathrm{k}}(f)$ as the percentage of power around the $f_{\mathrm{p}}(j, k)$ with respect to a reference interval $\Omega_{\mathrm{R}}(j, k)$ [11]. Then, a peaked-conditioned average spectra, $\bar{S}_{\mathrm{k}}(f)$, is obtained by averaging those $S_{\mathrm{j}, \mathrm{k}}(f)$ which are peaked enough:

$$
\bar{S}_{\mathrm{k}}(f)=\sum_{l=-L_{s}}^{L_{s}} \sum_{j=1}^{J} \chi_{j, k-l}^{A} \chi_{j, k-l}^{B} S_{j, k-l}(f),
$$




$$
\begin{aligned}
& P_{\mathfrak{M}}(n, m)=2 \cdot \sum_{l=-L+1}^{L-1}|h(l)|^{2} \cdot\left[\sum_{n^{\prime}=-N+1}^{N-1} g\left(n^{\prime}\right) a_{\mathfrak{M}}\left(n+n^{\prime}+l\right) a_{\mathfrak{M}}{ }^{*}\left(n+n^{\prime}-l\right)\right] \cdot e^{-j 2 l(m / M) \pi} \\
& m=-M+1 \ldots M
\end{aligned}
$$

where $L_{s}$ was set to 2 in order to average a maximum of 5 spectra as in [11], $J$ is the number of signals, and $\chi_{j, k-l}^{A}$ and $\chi_{j, k-l}^{B}$ are two criteria to consider whether the power spectrum $S_{j, k-l}(f)$ is peaked enough or not. These criteria allow to take part in the average only those $S_{\mathrm{j}, \mathrm{k}}(f)$ whose $P_{\mathrm{j}, \mathrm{k}}$ is above $85 \%$ (in this case $\chi_{j, k}^{A}=1$; otherwise, $\left.\chi_{j, k}^{A}=0\right)$ and its total power is greater than $95 \%$ of the maximum (in this case $\chi_{j, k}^{B}=1$; otherwise, $\chi_{j, k}^{B}=1$ ). So, the respiratory rate is estimated as:

$$
F_{\mathrm{R}}(k)=\arg \max _{f} \bar{S}_{\mathrm{k}}(f) .
$$

The respiratory rate is also used as an indicator to discard subjects. Those with $F_{\mathrm{R}}<0.15$ (8 subjects) or $F_{\mathrm{R}}>0.4$ ( 2 subjects) are discarded, because they could affect the ANS interpretation.

\subsection{Statistical analysis and classification}

A statistical analysis of the nine parameters obtained from ECG and the respiratory rate has been implemented in order to identify both states. Firstly, the Shapiro-Wilk test is applied to distinguish whether the signal has a normal distribution. When the normal distribution of the signal is verified, the t-Student test is applied. In other case, the Wilcoxon paired test is applied. A $p$-value $\leq 0.05$ define significance.

These tests are applied to each one of the 10 parameters extracted from the ECG signal during the BTA test and are normalized to the sum of the two states:

$$
X_{n B T A}^{i}=\frac{X_{B T A}^{i}}{X_{B T A}^{i}+X_{B L}^{i}}
$$

where $X$ is each one of the 10 parameters and $i=1 \ldots N_{s}$, being $N_{s}$ the number of subjects. For each parameter, subjects who exceed any of the following limits, in any of the two stages, are identified and eliminated:

$$
\begin{aligned}
\operatorname{lower}_{X} & =Q_{1}\left(X_{n B T A}^{i=1 . N_{s}}\right)-1.5 \cdot \operatorname{IQR}\left(X_{n B T A}^{i=1 . . N_{s}}\right) \\
\text { higher }_{X} & =Q_{3}\left(X_{n B T A}^{i=1 \ldots N_{s}}\right)+1.5 \cdot \operatorname{IQR}\left(X_{n B T A}^{i=1 . N_{s}}\right)
\end{aligned}
$$

The Ensemble Subspace Discriminant classifier has been used in order to classify states. Later on, it has been validated using Leave-One-Out cross-validation.

\section{Results and discussion}

Table 1 shows the values of all analyzed parameters for both stages and the results of the statistical analysis. These results show a significant decrease in the power of the HRV classical bands $\left(P_{\mathrm{HF}}\right.$ and $\left.P_{\mathrm{LF}}\right)$ during the BTA. Besides, a non-significant increase is shown in the normalized values of $P_{\mathrm{LFn}}$ and $R_{\mathrm{LFHF}}$. This decrease in the $P_{\mathrm{HF}}$ has been observed also in other works where subjects perform high level of attention demanding tasks [14], but their results show a non-significant increase in $P_{\mathrm{LF}}$, and a significant increase in $P_{\mathrm{LF}}$ and $R_{\mathrm{LF} H F}$. An increase in the $P_{\mathrm{LF}}$ and consequently in the sympathetic markers $\left(P_{\mathrm{LFn}}\right.$ and $\left.R_{\mathrm{LFHFF}}\right)$ is related to the activation of the sympathetic system, which occurs when the subject is exposed to various types of stressors (e.g. mental arithmetic, exams, reaction time, etc.) $[14,15]$. This behaviour is not experimented in our work due to BTA test has a low stress component, focused only on subject's attention, differently to [14], where the main goal lies in stress generation.

There is also a significant increase in respiratory rate and heart rate. The $F_{\mathrm{R}}$ has been identified in other works with significant differences between stress situations and high level of attentional states versus rest states [14]. The rest of temporal parameters show a significant decrease during the attention state.

A classifier with four of the most significant parameters $\left(P_{\mathrm{HF}}, H R M, p N N 50\right.$ and $\left.F_{\mathrm{R}}\right)$ has been trained an validated. The mean accuracy obtained in 25 repetitions is $75.91 \%$ (min: $73.26 \%$; max: $80.23 \%$ ).

\section{Conclusions}

This paper represents a first approximation to our ultimate goal: an automatic identifier of attentional states. Results suggest the use of only the ECG signal could be enough, as eight ECG-based parameters show significant differences between the two evaluated states; and with the use of only four of them, the mean accuracy is $75.91 \%$.

\section{Acknowledgements}

This work has been partially financed by Ministerio de Economía, Industria y Competitividad, FEDER, DGA and Centro Universitario de la Defensa througt the projects TEC2014-54143-P, TIN2014-53567-R, DGA T04-FSE, CUD2013-11, CUD2016-18 and UZCUD2016-TEC-03. The authors would like to thank Hospital General de la Defensa en Zaragoza, Regimiento de Pontoneros y Especialidades de Ingenieros $\mathrm{n}^{\mathrm{o}} 12$, Fuerzas Aeromóviles del Ejército de Tierra and Instituto Nacional de Técnica Aeroespacial Esteban Terradas for their valuable collaboration. 
Table 1. Values (mean \pm std) of time, frequency and respiration parameters at each stage (au. arbitrary units, ad. adimensional, nu. normalized units) and their normalization. Statistical differences are represented by: ${ }^{*}(p<0.05)$, ${ }^{* *}(p<0.001)$ and ${ }^{* * *}(p<0.0001)$. Final Size indicates final number of subjects used for each parameter.

\begin{tabular}{lcc||lcc}
\hline \hline Parameters & BL & BTA & BTA/(BL+BTA) & Final Size & Outliers \\
\hline$P_{\mathrm{LF}}\left(10^{-} 3 \mathrm{au}\right)$ & $4.21 \pm 7.31$ & $2.09 \pm 1.26$ & $0.43 \pm 0.19^{*}$ & 43 & 0 \\
$P_{\mathrm{HF}}\left(10^{-} 3 \mathrm{au}\right)$ & $1.87 \pm 2.10$ & $1.02 \pm 0.82$ & $0.41 \pm 0.16^{* *}$ & 40 & 3 \\
$P_{\mathrm{LFn}}(\mathrm{nu})$ & $0.66 \pm 0.18$ & $0.69 \pm 0.14$ & $0.52 \pm 0.06$ & 40 & 3 \\
$R_{\mathrm{LFHF}}(\mathrm{ad})$ & $3.12 \pm 3.31$ & $3.05 \pm 2.41$ & $0.55 \pm 0.18$ & 43 & 0 \\
$H R M($ beats/min $)$ & $68.96 \pm 10.87$ & $72.73 \pm 11.47$ & $0.51 \pm 0.02^{* * *}$ & 43 & 0 \\
$S D N N($ beats/min) & $68.46 \pm 29.45$ & $53.18 \pm 19.25$ & $0.44 \pm 0.09^{* *}$ & 43 & 0 \\
$S D S D($ beats/min$)$ & $52.83 \pm 32.80$ & $42.16 \pm 24.75$ & $0.45 \pm 0.08^{* *}$ & 41 & 2 \\
$R M S S D($ beats/min $)$ & $52.63 \pm 32.64$ & $42.03 \pm 24.64$ & $0.45 \pm 0.08^{* *}$ & 41 & 2 \\
$p N N 50(\%)$ & $26.54 \pm 20.46$ & $19.77 \pm 19.73$ & $0.36 \pm 0.19^{* * *}$ & 41 & 2 \\
$F_{\mathrm{R}}(\mathrm{Hz})$ & $0.25 \pm 0.05$ & $0.29 \pm 0.06$ & $0.54 \pm 0.04^{* * *}$ & 39 & 4 \\
\hline \hline
\end{tabular}

\section{References}

[1] Shin L, Shin P, Heckers S, Krangel T. Hippocampal function in posttraumatic stress disorder. Hippocampus 2004; 14:292-300.

[2] Thomas ML, Sing HC, Belenky G, Holcomb HH, Mayberg HS, Dannals RF, Wagner HN, Thorne DR, Popp KA, Rowland LM, Welsh AB, Balwinski SM, Redmond DP. Neural basis of alertness and cognitive performance impairments during sleepiness II. Effects of 48 and $72 \mathrm{~h}$ of sleep deprivation on waking human regional brain activity, 2003.

[3] Kahneman D. Attention and effort. New York, NY, USA: Prentice-Hall, 1973.

[4] Schretlen D, Bobholz JH, Brandt J. Development and psychometric properties of the brief test of attention. Clinical Neuropsychologist 1996;10(1):80-89. ISSN 1385-4046.

[5] TFESC, NASPE. Heart Rate Variability: Standards of Measurement, Physiological Interpretation, and Clinical Use, 1996.

[6] Sokas D, Gailius M, Marozas V. Diver physiology monitor and its graphical user interface. In Proceedings of International Scientific - Practical Conference, Virtual Instruments in Biomedicine. 2016; 5-9.

[7] Bailón R, Laouini G, Grao C, Orini M, Laguna P, Meste O. The integral pulse frequency modulation model with time-varying threshold: Application to heart rate variability analysis during exercise stress testing. Biomedical Engineering IEEE Transactions on March 2011;58(3):642-652. ISSN 0018-9294.

[8] Martinez JP, Almeida R, Olmos S, Rocha A, Laguna P. A wavelet-based ECG delineator: evaluation on standard databases. IEEE Transactions on Biomedical Engineering April 2004;51(4):570-581. ISSN 0018-9294.

[9] Mateo J, Laguna P. Analysis of heart rate variability in the presence of ectopic beats using the heart timing signal. IEEE Transactions on Biomedical Engineering 2003; 50(3):334-343.
[10] Bailón R, Garatachea N, de la Iglesia I, Casajús J, Laguna P. Influence of running stride frequency in heart rate variability analysis during treadmill exercise testing. Biomedical Engineering IEEE Transactions on July 2013;60(7):17961805. ISSN 0018-9294.

[11] Lázaro J, Alcaine A, Romero D, Gil E, Laguna P, Pueyo E, Bailón R. Electrocardiogram derived respiratory rate from qrs slopes and r-wave angle. Annals of Biomedical Engineering 2014;40(10):2072-2083.

[12] Pueyo E, Sörnmo L, Laguna P. QRS slopes for detection and characterization of myocardial ischemia. IEEE Transactions on Biomedical Engineering 2008;55(2):468477. ISSN 00189294.

[13] Romero D, Ringborn M, Laguna P, Pahlm O, Pueyo E. Depolarization changes during acute myocardial ischemia by evaluation of QRS slopes: Standard lead and vectorial approach. IEEE Transactions on Biomedical Engineering 2011;58(1):110-120. ISSN 00189294.

[14] Hernando A, Lazaro J, Gil E, Arza Valdes A, Garzon-Rey J, Lopez-Anton R, de la Camara C, Laguna P, Aguilo J, Bailón $R$. Inclusion of respiratory frequency information in heart rate variability analysis for stress assessment. IEEE Journal of Biomedical and Health Informatics 2016;1-1. ISSN 2168-2194.

[15] Berntson GG, Cacioppo JT, Fieldstone A. Illusions, arithmetic, and the bidirectional modulation of vagal control of the heart. Biological Psychology 1996;44:1-17. ISSN 03010511 .

Address for correspondence:

María Dolores Peláez Coca

Centro Universitario de la Defensa (CUD)

Academia General Militar (AGM)

Ctra. Huesca s/n, 50090 Zaragoza, Spain.

mdpelaez@unizar.es 